\title{
Effects of retinoid therapy on insulin sensitivity, lipid profile and circulating adipocytokines
}

\author{
S Corbetta, R Angioni, A Cattaneo ${ }^{1}$, P Beck-Peccoz and A Spada \\ Institute of Endocrine Sciences, University of Milan, Ospedale Maggiore IRCCS, Via F.Sforza, 35 20122, Milan, Italy and ${ }^{1}$ Unità Operativa di \\ Dermatologia 2, University of Milan, Ospedale Maggiore IRCCS, Milan, Italy
}

(Correspondence should be addressed to S Corbetta; Email: sabrina.corbetta@unimi.it)

\begin{abstract}
Objective: In vitro and in vivo models indicate that all-trans retinoic acids influence glucose and lipid metabolism. We aimed to evaluate the effects of chronic treatment with acitretin, an all-trans retinoic acid, on glucose metabolism, lipid profile and adiponectin and resistin levels.

Design: Ten normoglycemic, normolipemic patients affected with psoriasis vulgaris were studied before and after 1 and 3 months of oral treatment with $35 \mu \mathrm{g}$ of acitretin.

Methods: Glucose metabolism, lipid profile, and adiponectin and resistin levels were evaluated in basal conditions and after acitretin treatment. Ten healthy subjects matched for age, body mass index (BMI) and insulin sensitivity were studied as controls.

Results: One-month acitretin treatment reduced psoriasis activity, insulin sensitivity, evaluated as QUICKI values $(0.364 \pm 0.034$ versus $0.329 \pm 0.051 ; P<0.05)$ and HOMA-IR index $(1.53 \pm 0.73$ versus $2.59 \pm 1.41 ; P<0.05)$, and high-density lipoprotein (HDL)-cholesterol levels $(45.2 \pm 11.7$ versus $39.4 \pm 10.4 \mathrm{mg} / \mathrm{dl} ; P=0.01)$. The impairment in glucose and lipid homeostasis was transient and not associated to BMI variations. Adiponectin levels did not change during the treatment, while resistin levels, which were higher in untreated patients than in controls $(9.4 \pm 4.4$ versus $6.2 \pm 2.1 \mathrm{ng} / \mathrm{ml} ; P=0.05$ ), fell within the normal range after 1 and 3 months of therapy. The normalization of resistin levels occurred without significant changes in circulating tumor necrosis factor $\alpha$ $(\mathrm{TNF} \alpha)$ levels, which persisted elevated throughout the treatment.

Conclusions: Treatment with a low dose of acitretin induced a mild, transient reduction of insulin sensitivity and HDL-cholesterol levels that was not related to modifications of adiponectin, resistin and $\mathrm{TNF} \alpha$ levels. Although the role of resistin in humans remains elusive, the levels of this adipocytokine seem to be affected, at least in part, by retinoids.
\end{abstract}

European Journal of Endocrinology 154 83-86

\section{Introduction}

Retinoids, which include naturally occurring vitamin A, its metabolites and synthetic analogs, are physiological regulators of a number of essential biological processes, such as embryonic development, reproduction, metabolism, differentiation, proliferation and apoptosis (1). Retinoids are used clinically for the treatment of a number of dermatologic, hematopoietic and cancer diseases. Most of the effects of retinoids are mediated by retinoic acid receptors (RARs), which bind both all-trans retinoids and 9-cis retinoic acid, and the rexinoid receptors (RXRs), which bind 9-cis retinoic acid specifically (1).

Both retinoids and vitamin A status influence body adiposity by regulating the expression of adipogenic/lipogenic genes. In particular, retinoic acid, the carboxylic acid form of vitamin A, is a transcriptional activator of the genes encoding uncoupling proteins and evidence indicates that whole-body thermogenic capacity is related to the vitamin A status, with a low vitamin A status favoring increased fat deposition (2). All-trans retinoic acid is known to inhibit adipocyte differentiation (2). Though experimental evidence supports a role for retinoids in the regulation of glucose metabolism and adipose tissue physiology in animals, poor and inconsistent data are available in humans. In particular, etretinate therapy for psoriasis was reported to be associated with a reduction of glucose levels in response to a glucose load (3), whereas a recent study showed that isotretinoin treatment does not impair insulin sensitivity in healthy humans $(4,5)$.

In the present study we investigated the effects of chronic treatment with acitretin, an aromatic alltrans retinoic acid, on glucose metabolism, lipid profile and circulating levels of adiponectin and resistin. Adiponectin and resistin are two adipose tissue-specific cytokines that exert opposite effects on glucose metabolism, adiponectin acting as an insulin sensitizer and resistin as an insulin-resistance factor (6). Indeed, the expression of both adiponectin and resistin has 
been reported to be affected by retinoids in white adipose tissue of mice $(7,8)$, whereas in humans no data are available.

\section{Subjects and methods}

\section{Subjects}

Ten male patients, aged $41.0 \pm 9.1$ years, with mild to moderate psoriasis, defined by the Psoriasis Area and Severity Index (PASI; range 5.4-23.1), were recruited from the Ospedale Maggiore IRCCS Dermatological Unit, Milan, Italy (Table 1). Patients affected with overt diabetes, impaired glucose tolerance, impaired fasting glucose, severe dislipidemia, hypertension, cardiovascular diseases and familiarity for type 2 diabetes mellitus were excluded. All of the patients were drug free before to start the treatment with acitretin and none of them received medication other than oral acitretin and topical psoralen. Ten healthy subjects matched for age, sex, body mass index (BMI) and insulin sensitivity (homeostasis model assessment (HOMA)-IR and QUICKI values) were investigated under basal conditions as controls. The study protocol was approved by the Institutional Review Board and written informed consent was obtained from all patients.

\section{Methods}

The antropometric parameters of patients and controls were recorded at baseline (Table 1). Serum samples were collected after an overnight fasting and a resting period after the insertion of a venous catheter to determine serum glucose, insulin, total cholesterol, high-density lipoprotein (HDL)-cholesterol, low-density lipoprotein (LDL)-cholesterol, triglycerides, tumor necrosis factor $\alpha(\mathrm{TNF} \alpha)$, adiponectin and resistin levels. Serum glucose and lipid parameters were measured by routine assays. Circulating insulin, TNF $\alpha$ and adiponectin levels were measured with commercially available ELISA kits (Medgenix, Biosource Europe, Belgium; R\&D Systems, Minneapolis, MN, USA; B-Bridge International, San Jose, CA, USA). Circulating resistin levels were assayed with an ELISA kit provided by Alexis Italia

Table 1 Clinical and metabolic parameters of psoriasic patients $(n=10)$ at baseline and during acitretin treatment.

\begin{tabular}{lccc}
\hline & Basal & 1 month & 3 months \\
\hline $\mathrm{BMI}\left(\mathrm{kg} / \mathrm{m}^{2}\right)$ & $26.5 \pm 3.0$ & $26.4 \pm 2.9$ & $26.1 \pm 3.3$ \\
Glucose $(\mathrm{mmol} / \mathrm{l})$ & $4.96 \pm 0.81$ & $5.05 \pm 0.70$ & $5.11 \pm 0.78$ \\
Insulin $(\mu \mathrm{U} / \mathrm{ml})$ & $6.7 \pm 2.6$ & $11.6 \pm 6.5^{*}$ & $9.0 \pm 6.6$ \\
HOMA-IR & $1.53 \pm 0.73$ & $2.59 \pm 1.41^{*}$ & $2.04 \pm 1.68$ \\
QUICKI & $0.364 \pm 0.034$ & $0.329 \pm 0.051^{*}$ & $0.361 \pm 0.041$ \\
Total cholesterol $(\mathrm{mg} / \mathrm{dl})$ & $184.3 \pm 46.4$ & $182.6 \pm 48.1$ & $184.5 \pm 42.6$ \\
HDL-cholesterol $(\mathrm{mg} / \mathrm{dl})$ & $45.2 \pm 11.7$ & $39.4 \pm 10.4^{*}$ & $43.0 \pm 14.4$ \\
Triglycerides $(\mathrm{mg} / \mathrm{dl})$ & $107.2 \pm 48.0$ & $118.0 \pm 47.3$ & $114.2 \pm 34.9$ \\
\hline$* P<0.05$ versus basal. & & &
\end{tabular}

* $P<0.05$ versus basal.
Vinci-Biochem (Vinci, Florence, Italy), whose intra- and inter-assay coefficients of variation were 3.4 and $6.9 \%$, respectively, with a low detection limit of $0.2 \mathrm{ng} / \mathrm{ml}$ and no detectable cross-reactivity to other cytokines in human serum. Insulin resistance was calculated using the HOMA method and insulin sensitivity using the QUICKI index. Patients were revaluated after 1 and 3 months of oral treatment with $35 \mu \mathrm{g}$ /day acitretin.

\section{Statistical analysis}

Continuous variables were summarized as means \pm S.D. Comparisons among groups of different normally distributed parameters were performed with Student's $t$ test for unpaired data. Multiple measurements in the same patient were tested using one-way analysis of variance (ANOVA). Relationships between two variables were studied with linear regression. Statistical analyses were performed with Prism 4 software. A significance level of $5 \%$ was used throughout.

\section{Results}

\section{Effects of acitretin treatment on PASI index and body weight}

After 1 month of therapy with acitretin (35 $\mu \mathrm{g} /$ day) all patients showed a significant improvement of PASI index, with the severity of psoriasis ameliorating further after 3 months of treatment (Fig. 1). During the treatment, BMI values did not vary significantly with respect to the baseline conditions (Table 1).

\section{Effects of acitretin treatment on insulin sensitivity and lipid profile}

As reported in Table 1, 1-month treatment induced a slight but significant increase in serum insulin and HOMA-IR levels, while fasting serum glucose levels remained unchanged. Accordingly, calculated QUICKI values were significantly reduced (Table 1). These differences in insulin sensitivity were transient as they were abolished after 3 months. Acitretin treatment did not affect serum total cholesterol, LDL-cholesterol and triglycerides at 1 or 3 months of therapy. Conversely, serum HDL-cholesterol levels were significantly reduced by 1 month of therapy, and returned to baseline after 3 months (Table 1).

\section{Effects of acitretin treatment on circulating adiponectin, resistin and TNF $\alpha$ levels}

Serum adiponectin levels in psoriasic patients did not differ from those observed in healthy control subjects and did not vary during acitretin treatment. By contrast, psoriasic patients showed circulating resistin levels higher than those observed in control subjects $(9.4 \pm 4.4$ versus $6.2 \pm 2.1 \mathrm{ng} / \mathrm{ml}$ respectively; $P<0.05$ ), although 


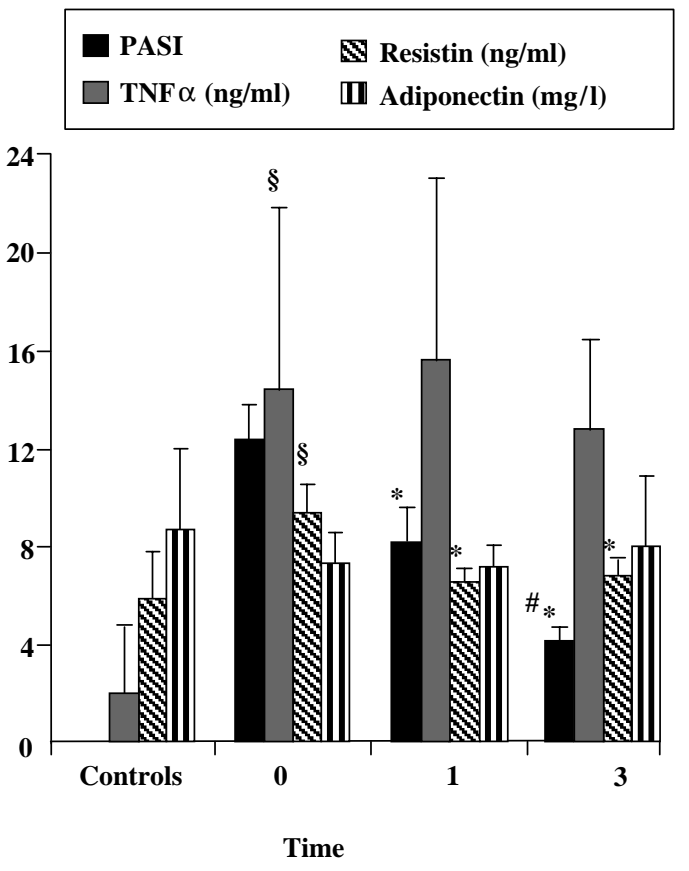

Figure 1 Effect of 1 and 3 months of acitretin treatment on psoriasis activity evaluated by PASI index and circulating TNF $\alpha$, adiponectin and resistin levels. Data are presented as means \pm S.D. $\S P<0.05$ versus controls; ${ }^{*} P<0.05$ versus 0 month; $\# P<0.05$ versus 1 month.

patients and controls had similar HOMA-IR (1.53 \pm 0.73 versus $2.80 \pm 1.80)$ and QUICKI $(0.364 \pm 0.034$ versus $0.331 \pm 0.059)$ values. Resistin levels were significantly reduced after 1 month of treatment (from 9.4 \pm 4.4 to $6.48 \pm 2.6 \mathrm{ng} / \mathrm{ml} ; P=0.002$ ), this reduction being maintained after 3 months (Fig. 1). Under basal conditions circulating TNF $\alpha$ levels were definitely elevated in three patients and were within the normal range in the remaining seven. TNF $\alpha$ levels did not correlate with the PASI index and did not vary after 1 or 3 months of therapy.

Both at baseline and under acitretin treatment, no significant correlation between serum $\mathrm{TNF} \alpha$, resistin and adiponectin levels and BMI, insulin, HOMA-IR, QUICKI, total cholesterol, LDL-cholesterol, HDL-cholesterol and tryglicerides was observed in psoriasic patients.

\section{Discussion}

This prospective study confirmed that chronic administration of retinoids may influence lipid metabolism and provided new evidence for a role of these agents in the regulation of glucose homeostasis and adipose tissuespecific cytokines in humans. The effects of low doses of acitretin were evaluated in an homogenous cohort of non-diabetic, normoglycemic and non-insulin-resistant patients affected with psoriasis and in good condition, who provided a model for the evaluation of drug-induced metabolic changes that was more suitable than hematopoietic and solid malignancies (9). Acitretin caused a transient impairment of insulin sensitivity, as indicated by the increases in insulin levels and HOMA-IR indices and the decreases in QUICKI values, which remained within the normal ranges. Although these subtle changes in glucose homeostasis were not explored using glucose tolerance tests, our data are in agreement with in vitro evidence showing that retinoid-induced expression of proteins is involved in glucose metabolism and signal recognition for insulin secretion. In particular it has been shown that alltrans retinoic acid enhanced phosphoenolpyruvate carboxykinase (PEPCK) gene transcription and glucose production in hepatocytes as well as insulin release from pancreatic $\beta$-cells after short-term exposure $(48 \mathrm{~h})(10,11)$. A few clinical studies have been performed in small series of patients that were heterogeneous for vitamin A-derivative therapy, time of treatment and insulin-sensitivity evaluation. Nonetheless, long-term treatment (20 weeks) with etretinate was reported to improve glucose tolerance in diabetic subjects (3), whereas two studies in healthy subjects reported no effect or improvement of insulin sensitivity during treatment with vitamin A derivatives. However, it is worth noting that these data were obtained after a short-term (5-7 days) treatment using different protocols for the assessment of glucose metabolism. Indeed, our results were in agreement with data from a recent study finding a reduction in insulin sensitivity evaluated by the euglycemic/hyperinsulinemic clamp in otherwise healthy men with acne who were treated orally for 5 months with 13-cis retinoic acid (12).

Retinoid therapy is known to be associated with hypertriglyceridemia and often with low HDL-cholesterol $(13,14)$. Significant alterations in serum triglycerides levels were not detected in this series, likely due to the low doses of acitretin used. By contrast, a significant decrease in serum HDL-cholesterol was observed after 1 month of treatment. This finding is consistent with a previous study reporting down-regulation of the expression of apolipoprotein A-I mRNA in rat hepatocytes, a major component of HDL-cholesterol, by alltrans retinoids (15). Both the increase in insulin levels and the decrease in HDL-cholesterol were transient, suggesting that in the long term other metabolic regulatory pathways may override the subtle alterations in glucose and lipid metabolism induced by retinoids.

We tested the hypothesis that the transient impairment of glucose and lipid homeostasis might be sustained by the effects of retinoids on adipocyte function, and particularly on the production of resistin and adiponectin, two adipose-specific hormones involved in obesity, insulin resistance and/or metabolic and cardiovascular risk factors $(6,16)$. In fact, it has been reported that retinoic acid and vitamin A reduced the expression of both resistin and adiponectin in rodent white adipocytes $(7,8)$. In contrast to these 
observations, the present study seems to exclude a direct involvement of these adipocytokines in the changes in glucose and lipid homeostasis induced by retinoids. In fact, the transient impairment of insulin sensitivity was associated with neither a decrease of adiponectin levels nor an increase in resistin levels.

Finally we investigated the possible influence of changes in $\mathrm{TNF} \alpha$, a cytokine involved in the pathogenesis of psoriasis that may increase resistin expression in peripheral blood monocytes $(17,18)$, on resistin levels. The study showed a discordant pattern of TNF $\alpha$ and resistin secretion since serum $\mathrm{TNF} \alpha$ levels were definitely high only in a minority of patients while resistin levels were elevated in all patients and normalization of resistin was associated with any changes in $\mathrm{TNF} \alpha$ levels. Taken together these observations suggest a direct effect of acitretin on resistin expression from monocytes/macrophages and/or adipose tissue, in analogy with previous data obtained in mice (7). Admittedly, it is not possible to rule out from our study the involvement of cytokines other than $\mathrm{TNF} \alpha$ in this phenomenon.

In conclusion, we have demonstrated that treatment with a low dose of acitretin induced a mild and transient reduction of insulin sensitivity and HDL-cholesterol levels which was not related to modifications of adiponectin and resistin levels. Though the actual role of resistin in humans remains elusive, circulating levels of this adipocytokine seem to be affected, at least in part, by retinoids.

\section{References}

1 Aranda A \& Pascual A. Nuclear hormone receptors and gene expression. Physiological Reviews 200181 1269-1304.

2 Bonet ML, Ribot J, Felipe F \& Palou A. Vitamin A and the regulation of fat reverses. Cellular and Molecular Life Sciences 2003 $601311-1321$

3 Ellis CN, Kang S, Vinik AI, Grekin RC, Cunningham WJ \& Voorhees JJ. Glucose and insulin responses are improved in patients with psoriasis during therapy with etretinate. Archives of Dermatology $1987 \mathbf{1 2 3}$ 471-475.

4 Stoll D, Binnert C, Mooser V \& Tappy L. Short-term administration of isotretinoin elevates plasma triglyceride concentrations without affecting insulin sensitivity in healthy humans. Metabolism $2004 \mathbf{5 3} 4-10$.

5 Hartmann D, Forgo I, Dubach UC \& Hennes U. Effect of acitretin on the response to an intravenous glucose tolerance test in healthy volunteers. European Journal of Clinical Pharmacology $199242523-528$.
6 Fasshauer M \& Paschke R. Regulation of adipocytokines and insulin resistance. Diabetologia 200346 1594-1603.

7 Felipe F, Bonet ML, Ribot J \& Palou A. Modulation of resistin expression by retinoic acid and vitamin A status. Diabetes 2004 $53882-889$.

8 Zhang Y, Matheny M, Zolotukhin S, Tumer N \& Scarpace PJ. Regulation of adiponectin and leptin gene expression in white and brown adipose tissues: influence of beta3-adrenergic agonists, retinoic acid, leptin and fasting. Biochimica et Biophysica Acta $20021584115-122$.

9 Freemantle SJ, Spinella MJ \& Dmitrovsky E. Retinoids in cancer therapy and chemoprevention: promise meets resistance. Oncogene 200322 7305-7315.

10 Lee MY, Jung CH, Lee K, Choi YH, Hong S \& Cheong J. Activating transcription factor- 2 mediates transcriptional regulation of gluconeogenic gene phosphoenolpyruvate carboxykinase by retinoic acid. Diabetes 200251 3400-3407.

11 Cabrera Valladares G, German MS, Matschinsky FM, Wang J \& Fernandez Mejia C. Effect of retinoic acid on glucokinase activity and gene expression and on insulin secretion in primary cultures of pancreatic islets. Endocrinology 1999140 3091-3096.

12 Koistinen HA, Remitz A, Gilling H, Miettinen TA, Koivisto VA \& Ebeling P. Dyslipidemia and a reversible decrease in insulin sensitivity induced by therapy with 13-cis-retinoic acid. Diabetes/Metabolism Research and Reviews 200117 391-395.

13 Zech LA, Gross EG, Peck GL \& Brewer HB. Changes in plasma cholesterol and triglyceride levels after treatment with oral isotretinoin. A prospective study. Archives of Dermatology 1983119 987-993.

14 Matel-Teeuwisse AK, Kloosterman JM, Maitland-van der Zee AH, Klungel OH, Porsius AJ \& de Boer A. Drug-induced lipid changes: a review of the unintended effects of some commonly used drugs on serum lipid levels. Drug Safety 200124 1017-1018.

15 Berthou L, Staels B, Saldicco I, Berthelot K, Casey J, Fruchart JC, Denefle P \& Branellec D. Opposite in vitro and in vivo regulation of hepatic apolipoprotein A-I gene expression by retinoic acid. Absence of effects on apolipoprotein A-II gene expression. Arteriosclerosis, Thrombosis and Vascular Biology 199414 1657-1664.

16 Shetty GK, Economides PA, Horton ES, Mantzoros CS \& Veves A. Circulating adiponectin and resistin levels in relation to metabolic factors, inflammatory markers and vascular reactivity in diabetic patients and subjects at risk for diabetes. Diabetes Care 200427 $2450-2457$.

17 Schottelius AJ, Moldawer LL, Dinarello CA, Asadullah K, Sterry W \& Edwards CK III. Biology of tumor necrosis factor-alpha - implications for psoriasis. Experimental Dermatology 200413 193-222.

18 Kaser S, Kaser A, Sandhofer A, Ebenbichler CF, Tilg H \& Patsch JR. Resistin messenger-RNA expression is increased by proinflammatory cytochines in vitro. Biochemical and Biophysical Research Communications $2003309286-290$.

Received 13 June 2005

Accepted 16 September 2005 